\title{
MISE EN EVIDENCE D'UN FLUX GÉNIQUE ENTRE POPULATIONS NATURELLES DE TRUITE FARIO ET SOUCHE DE REPEUPLEMENT DANS DEUX RIVIËRES DE CORSE
}

\author{
R. GUYOMARD, Francine KRIEG
}

Laboratoire de Génétique des Poissons

I.N.R.A., 78350 JOUY-en-JOSAS, France

\section{RÉSUMÉ}

La comparaison d'échantillons de truite fario (Salmo trutta L.) prélevés dans différents bassins hydrographiques de Corse avec la souche de pisciculture utilisée pour le repeuplement dans cette zone a permis de définir des variants électrophorétiques caractéristiques de la souche domestique. L'absence de ces allèles dans les échantillons prélevés dans les rivières non sujettes au repeuplement et leur présence simultanée dans les échantillons provenant de cours d'eau, où les repeuplements sont régulièrement réalisés, mettent clairement en évidence une modification de la structure génétique des stocks locaux par les sujets de pisciculture déversés. L'analyse de la structure génotypique des échantillons génétiquement "contaminés" indique, en outre, qu'il n'y a pas d'isolement reproducteur total, en milieu naturel, entre souche de pisciculture et populations naturelles de Corse.

\section{EVIDENCE OF GENE FLOW BETWEEN WILD BROWN TROUT AND RESTOCKING STRAIN IN TWO CORSICAN DRAINAGES}

\section{SUMMARY}

An electrophoretic analysis of brown trout samples from different Corsican drainages and of the domesticated strain usually introduced in some of these drainages enabled us to define hatchery specific variants. These variants were not found in rivers which never received any planting. By contrast, they appear simultaneously in rivers where hatchery strains were continuously stocked, clearly indicating that genetic "contaminations" by restocking occurred in these drainages. Furthermore, the genotype analysis of the native samples showed that there is no complete reproductive isolation in the wild between the Corsican populations and the introduced strain.

\section{INTRODUCTION}

La truite fario fait l'objet, dans la majorité des rivières françaises, de repeuplements dont on peut retenir les caractéristiques suivantes (CHEVASSUS et GUYOMARD, 1983):

- (1) Les sujets de repeuplement sont issus de souches entretenues en pisciculture depuis plusieurs générations (dans de rares cas, des mâles sauvages prélevés localement sont utilisés comme géniteurs).

- (2) $80 \%$, au moins, des souches de repeuplement sont issues d'une dizaine de piscicultures à large diffusion. Les souches maintenues dans ces piscicultures-pivots semblent avoir ellesmêmes, dans certains cas, une origine commune.

- (3) II y a peu de rapport entre la zone d'origine ou de production des stocks de repeuplement et leurs zones d'utilisation. Certaines productions "couvrent" toute la France.

II est facile d'imaginer, à partir de ces trois caractéristiques, les conséquences à long terme du repeuplement :

- un remplacement des stocks locaux par des stocks domestiques qui peuvent être génétiquement très différents des premiers (conséquences de (1) et (3)) et inadaptés aux conditions locales (conséquences de (1) et (3)):

- une réduction de la diversité génétique de l'espèce (conséquences de (1) et (2)).

Une étude comparative de la diversité génétique des populations naturelles et domestiques a donc été entreprise pour mesurer l'ampleur des risques que nous venons de mentionner. 
Cette diversité génétique a été estimée à partir de variations des systèmes enzymatiques ou protéïques, mises en évidence par électrophorèse. Contrairement à celles des caractères classiquement utilisés pour décrire les différences entre populations (caractères morphologiques, méristiques ou de coloration de la robe), les variations des systèmes enzymatiques présentent l'avantage d'avoir un déterminisme génétique simple et d'être très peu affectées par les différences environnementales. Elle peuvent doncêtre directement examinées sur des populations prélevées dans des piscicultures ou rivières différentes et facilement traduites en termes de fréquences génotypiques et géniques.

Les résultats obtenus jusqu'à présent (GUYOMARD et KRIEG, 1983; KRIEG et GUYOMARD. 1983, 1985; KRIEG, 1984) ont permis d'établir qu'il existe une importante différenciation entre populations naturelles. Les populations méditerranéennes et atlantiques constituent deux groupes génétiquement très différenciés. Au sein de ces deux groupes, il subsiste une forte variabilité génétique interpopulations. Par contre, les populations de pisciculture sont génétiquement très proches les unes des autres, peu différentes de certaines populations naturelles normandes et nord-bretonnes, mais très distinctes des populations méditerranéennes et du sud-ouest de la Bretagne. L'utilisation intensive des souches de repeuplement peut donc aboutir, à terme, à l'élimination de stocks naturels très originaux. On peut estimer à environ $50 \%$ la perte de variabilité de l'espèce qui résulterait du remplacement des populations naturelles, que nous avons étudiées, par des souches d'élevage. Dans cet article, nous présentons des résultats qui mettent en évidence l'existence d'une "contamination génétique", due au repeuplement, de certaines populations naturelles de Corse.

\section{MATÉRIEL ET MÉTHODES}

\section{Echantillons étudiés :}

Le tableau no 1 donne la localisation géographique et certaines caractéristiques des échantillons étudiés. Les informations relatives au repeuplement darıs les rivières et sites concernés par cette étude nous ont été fournies par le Parc Naturel Régional de Corse.

\begin{tabular}{|c|c|c|}
\hline ORIGINE DU PRELEVEMENT & TAILLE DE L'ECHANTILLON & OBSERVAT IONS \\
\hline Rivière Aitone & 20 & Pas de repeuplement \\
\hline Rivière Travo & 10 & " \\
\hline $\begin{array}{c}\text { St-Antoine (rivière } \\
\text { Rizzanèse) }\end{array}$ & 18 & $\begin{array}{l}\text { Repeuplement par souche } \\
\text { Etrun }\end{array}$ \\
\hline Chiuvone Crivière & 23 & $"$ \\
\hline Asinao Crivière & 9 & $"$ \\
\hline Ste-Lucie Crivière & 17 & $"$ \\
\hline Rivière Solenzara & 15 & $"$ \\
\hline Pisciculture d'Etrun & 50 & $\begin{array}{l}\text { Seule souche de pisciculture } \\
\text { utilisée en Corse pour le } \\
\text { repeuplement }\end{array}$ \\
\hline
\end{tabular}

Tableau 1 : Localisation géographique et caractéristiques des échantillons examinés dans cette étude.

Table 1 : Geographical localization and characteristics of the samples examined in this study.

\section{Méthodes électrophorétiques :}

Les conditions d'électrophorèse et de révélations des systèmes enzymatiques ont été détaillées dans des articles précédents (GUYOMARD et KRIEG, 1983; KRIEG, 1984). Les systèmes enzymatiques étudiés ont été décrits par KRIEG (1984), et KRIEG et GUYOMARD (1985). Dans cet article, seuls seront pris en considération les systèmes polymorphes. Le déterminisme génétique de ces systèmes ainsi que la nature génétique de leurs variations électrophorétiques ont été démontrés pour la plupart d'entre eux (GUYOMARD et KRIEG, 1983 ; KRIEG, 1984 ; GUYOMARD, sous presse). 


\section{RESULTATS}

Le tableau no 2 présente les fréquences alléliques observées dans les 8 échantillons étudiés. Une comparaison des fréquences observées, d'une part, dans la souche de repeuplement (Etrun) et, d'autre part, dans les deux populations ne faisant l'objet d'aucun repeuplement (Aitone et Travo), met en évidence des allèles caractéristiques de la souche d'élevage (absents dans les deux populations corses) : Aat-2 (130), Aat-4 (65), Agp-2 (50), Cpk-1 (125), Fdp-1 (100), Fum-1,2 (115), (130) et (140), Idh-3 (200), Ldh-5 (100), Mdh-2 (200), Mdh-3 (75), Pmi-2 (105), Sdh-1 (-100). Certains d'entre eux atteignent des fréquences très élevées dans la souche de pisciculture (1. pour Ldh-5 (100); 0,86 pour Fdp-1 (100) : 0,61 pour Pmi-2 (105)). Dans le cas de Fum-1,2, il est impossible d'attribuer les variations observées à l'un ou l'autre des deux locus (Fum-1 et 2) et nous avons conc admis que chacun d'eux possédait les trois variants à des fréquences identiques. Dans la suite du texte, nous traiterons Fum-1,2 (115). Fum-1,2 (130) et Fum-1,2 (140) comme un seul allèle.

\begin{tabular}{|c|c|c|c|c|c|c|c|c|c|}
\hline & & ETRUN & AITONE & TRAVO & SI-ANTOINE & CHIJVONE & STE-LUCIE & As Imio & SOLENZARA \\
\hline \multirow[t]{2}{*}{ Aat -2} & 100 & 0.90 & 1.00 & 1.08 & 1.00 & 1.00 & 1.00 & 1.00 & 1.00 \\
\hline & 130 & 0.10 & 0.00 & 0.00 & 0.00 & 0.00 & 0.00 & 0.00 & 0.00 \\
\hline \multirow[t]{2}{*}{ Aat -4} & 100 & 0.81 & 1.00 & 1.00 & 0.95 & n.e. & 1.00 & 1.00 & 0.97 \\
\hline & 65 & 0.19 & 0.00 & 0.00 & 0.05 & & 0.00 & 0.00 & 0.03 \\
\hline \multirow[t]{2}{*}{ Agp -2} & 100 & 0.93 & 1.00 & 1.00 & $0.95(1.00)^{*}$ & 1.00 & 1.00 & 1.00 & 1.00 \\
\hline & so & 0.07 & 0.00 & 0.00 & $0.05(0.00)$ & 0.00 & 0.00 & 0.00 & 0.00 \\
\hline \multirow[t]{2}{*}{$C_{p k}-2$} & 100 & 0.85 & 1.00 & 1.00 & $0.95(1.00)$ & 1.00 & 1.00 & 1.00 & 1.00 \\
\hline & 125 & 0.15 & 0.00 & 0.00 & $0.05(0.00)$ & 0.00 & 0.00 & 0.00 & 0.00 \\
\hline \multirow[t]{3}{*}{$f d p-1$} & 100 & 0.86 & 0.00 & 0.00 & 0.10 & n.e. & 0.00 & 0.50 & 0.17 \\
\hline & 150 & 0.14 & 1.00 & 1.00 & 0.90 & & 1.00 & 0.50 & 0.73 \\
\hline & 80 & 0.00 & $0 . n 0$ & 0.00 & 0.00 & & 0.00 & 0.00 & 0.10 \\
\hline & 0.57 & 1.00 & 1.00 & 0.95 & 0.98 & 0.99 & 0.77 & 0.97 \\
\hline \multicolumn{2}{|c|}{$\begin{array}{r}\text { fum }-7-2100 \\
115\end{array}$} & 0.14 & 0.00 & 0.00 & 0.04 & 0.00 & 0.00 & 0.17 & 0.03 \\
\hline \multirow{2}{*}{\multicolumn{2}{|c|}{$\begin{array}{l}130 \\
140\end{array}$}} & 0.15 & 0.00 & 0.00 & 0.01 & 0.02 & 0.00 & 0,03 & 0.00 \\
\hline & & 0.14 & 0.00 & 0.00 & 0.00 & 0.00 & 0.01 & 0.03 & 0.00 \\
\hline \multirow[t]{2}{*}{$\operatorname{Idn}-3$} & 100 & 0.88 & 1.00 & 1.00 & 1.00 & n.e. & 1.00 & 1.00 & 1.00 \\
\hline & 200 & 0.12 & 0.00 & 0.00 & 0.00 & & 0.00 & 0.00 & 0.00 \\
\hline \multirow[t]{2}{*}{ Idh-4 } & 100 & 1.00 & 1.00 & 1.00 & 1.00 & n.e. & 1.00 & 1.00 & 0.33 \\
\hline & 80 & 0.00 & 0.00 & 0.00 & 0.00 & & 0.00 & 0.00 & 0.67 \\
\hline \multirow[t]{2}{*}{ Lan-3 } & 100 & .1 .00 & 1.00 & 0.00 & 0.11 & 0.50 & 0.38 & 0.17 & 0.63 \\
\hline & 63 & 0.00 & 0.00 & 1.00 & 0.89 & 0.50 & 0.62 & 0.83 & 0.37 \\
\hline \multirow[t]{2}{*}{ Ldh-5 } & 100 & 1.00 & 0.00 & 0.00 & $0.14(0.08)$ & 0.04 & 0.00 & 0.33 & 0.00 \\
\hline & 105 & $0.0 \mathrm{Q}$. & 1.00 & 1.00 & $0.86(0.92)$ & 0.96 & 1.00 & 0.66 & 1.00 \\
\hline \multirow[t]{3}{*}{$\operatorname{man}-2$} & 100 & 0.79 & 1.00 & 0.40 & $c .92$ & 0.15 & 0.41 & 1.50 & c.?! \\
\hline & 200 & 0.21 & 0.00 & 0.00 & 0.00 & 0.00 & 0.00 & 0.00 & 0.13 \\
\hline & 0 & 0.00 & 0.00 & 1.00 & 0.08 & 0.85 & 0.59 & 0.00 & 0.13 \\
\hline \multirow[t]{3}{*}{ Moh-3 } & 100 & 0.70 & 0.60 & 1.00 & 0.98 & 0.96 & 0.91 & 0.94 & 1.00 \\
\hline & 75 & 0.30 & 0.00 & 0.00 & 0.02 & 0.04 & 0.09 & 0.08 & 0.00 \\
\hline & 60 & & 0.40 & 0.00 & 0.00 & 0.00 & 0.00 & 0.00 & 0.00 \\
\hline \multirow[t]{2}{*}{ Me -1} & 100 & 1.00 & 0.77 & 0.00 & 1.00 & 0.59 & 1.00 & 1.00 & 0.90 \\
\hline & 150 & 0.00 & 0.23 & 1.00 & 0.00 & 0.41 & 0.00 & 0.00 & 0.10 \\
\hline \multirow[t]{2}{*}{$P_{\square i-2}$} & 100 & 0.39 & 1.00 & 1.00 & 0.95 & 0.96 & 1.00 & 0.66 & 0,93 \\
\hline & 105 & 0.01 & 0.00 & 0.00 & 0.05 & 0.04 & 0.00 & 0.33 & 0.07 \\
\hline \multirow[t]{2}{*}{$S d h-1$} & 100 & 0.25 & 0.00 & 0.00 & 0.00 & n.e. & 0.00 & 0.00 & 0.03 \\
\hline & 0 & 0.75 & 1.00 & 1.00 & 1.00 & & 1.00 & 1.00 & 0.97 \\
\hline \multirow[t]{2}{*}{$5 d h-2$} & 100 & 1.00 & 1.00 & 0.00 & 0.08 & n.e. & 0.00 & 0.33 & 0.93 \\
\hline & 170 & 0.00 & 0.00 & 1.00 & 0.92 & & 1.00 & 0.66 & 0.07 \\
\hline \multirow[t]{3}{*}{ If } & 100 & 1.00 & 0.00 & 0.00 & n.e: & n.e. & n.e. & n.e. & n.e. \\
\hline & 99 & 0.00 & 0.63 & $1 . \mathrm{m}$ & & & & & \\
\hline & 80 & 0.00 & 0.37 & 0.00 & & & & & \\
\hline
\end{tabular}

- entre parenthèses : fréquences recalculées en éliminant l'individu provenant de la souche d'élevage.

(voir texte).

n.e. : non átudié.

Tableau 2 : Fréquences alléliques dans les échantillons étudiés.

Table 2 : Allele frequencies in the samples studied. 
Tout échantillon issu d'une population corse "génétiquement contaminée" par la souche Etrun se caractérisera donc par la présence simultanée des allèles spécifiques de cette souche. Sil'introduction est récente, on peut s'attendre à ce que les fréquences de ces allèles, dans la population contaminée, soient proportionnelles à celles observées dans la souche domestique. Au cours des premières générations (au moins jusqu'à la deuxième), des déséquilibres de liaison (par exemple, des associations préférentielles d'allèles de pisciculture chez un même individu) devraient être observés.

Enfin, toute "contamination" importante devrait se traduire par un accroissement notable de la variabilité génétique de la population naturelle.

Les deux premiers phénomènes apparaissent très clairement dans les échantillons prélevés sur le Rizzanèse (Asinao, St-Antoine, Chiuvone et Ste-Lucie). Les quatre allèles les plus discriminants de la souche domestique, Ldh-5 (100), Fdp-1 (100), Pmi-3 (105) et Fum-1,2 (115+130+140) sont observés dans 3 des 4 échantillons collectés dans cette rivière. Leurs fréquences relatives ne sont pas indépendantes. Pratiquement absents sur le site de Ste-Lucie, ils apparaissent simultanément à des fréquences faibles sur ceux de Chiuvone et St-Antoine, et moyennes sur celui d'Asinao (figure 1).

\section{ETRUN}

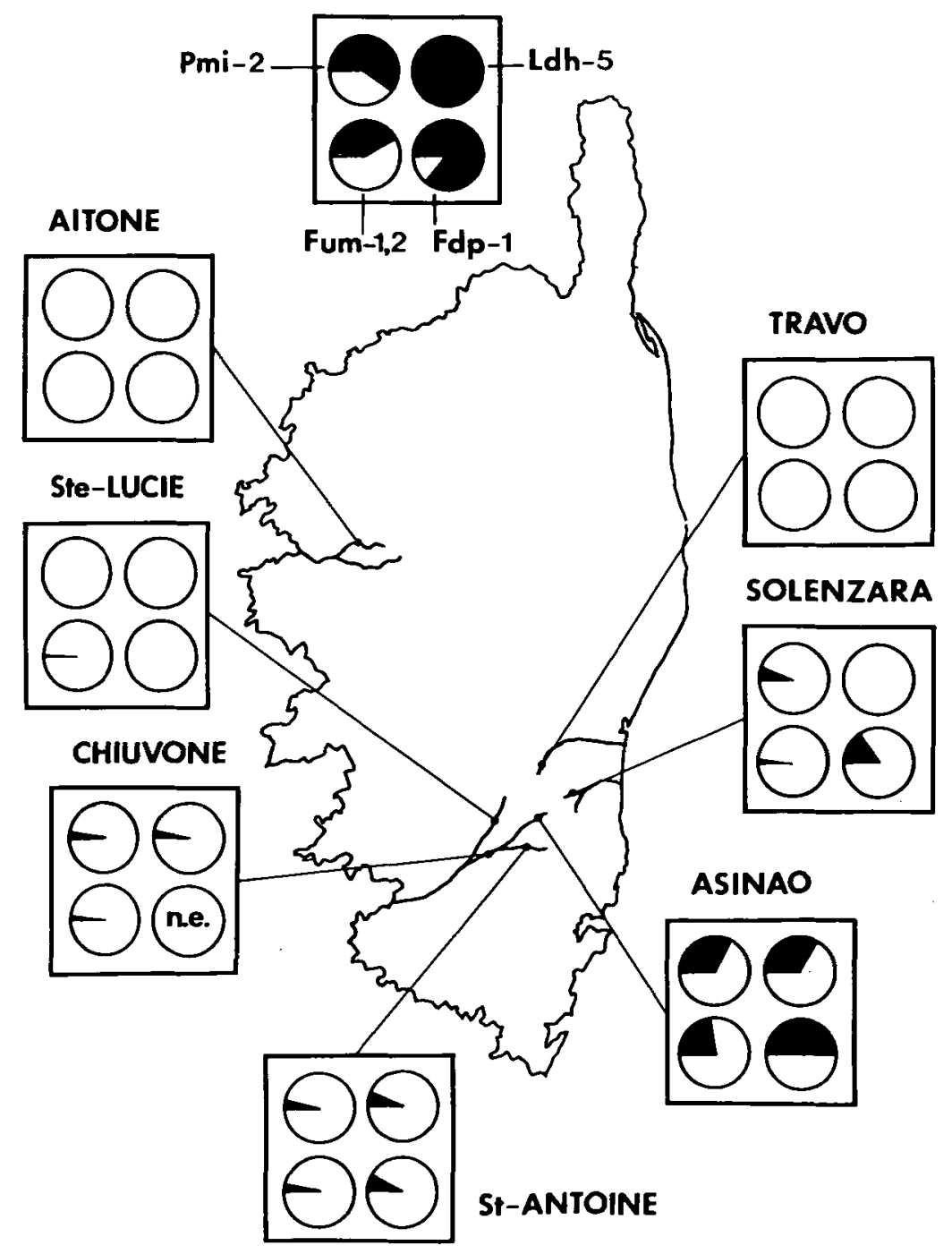

Figure 1 : Fréquences des quatre allèles "domestiques" les plus discriminants (secteurs noirs) dans les huit échantillons domestique et corses étudiés.

Figure 1 : Frequencies of the four most discriminating "hatchery-alleles" (black sectors) in the eight samples studied. 
Dans ce dernier site, la présence de ces quatre allèles se traduit par un accroissement notable de la variabilité intrapopulation (6.8 contre 3.5 en moyenne, figure 2 ). Ces premières constatations indiquent donc très clairement la présence de gènes d'origine domestique dans la population du Rizzanèse. La fréquence moyenne de ces gènes peut être estimée à $0 \%, 5 \%, 10 \%$ et $50 \%$ respectivement dans les échantillons Ste-Lucie, Chiuvone, St-Antoine et Asinao. L'analyse cladistique (HENNIG, 1966) appliquée à nos résultats renforce cette conclusion. Cette approche établit une distinction très nette entre formes dérivées qui seules permettent de retracer les relations généalogiques entre groupes de populations ou d'espèces et les formes ancestrales qui n'ont pas de valeur phylogénétique. Les formes dérivées sont déterminées par rapport aux formes ancestrales qui sont celles que I'on retrouve à la fois chez l'espèce étudiée et celle qui lui est 'a plus proche, en l'occurence, Je saumon atlantique. Ainsi, les quatre électromorphes Fdp-1 (100), Ldh-5 (100), Fum-1,2(115+130+140) et Pmi-2 (105) constituent des formes dérivées caractéristiques des populations domestiques et de certains stocks des rivières tributaires de l'océan Atlantique. Une même forme dérivée ne pouvant apparaître indépendamment dans deux populations différentes, selon les principes de la cladistique, il faut donc admettre que leur présence simultanée dans les populations domestiques et corses est due à une migration, c'est-a-dire une introduction par repeuplement, après apparition dans le groupe des populations dont sont issues les souches d'élevage. Par contre, l'allèle Mdh-3(75) est observé à la fois chez la truite fario et le saumon atlantique; il s'agit donc d'une forme ancestrale qui a pu se maintenir dans certaines populations. Sa présence dans le Rizzanèse, même si elle est attendue, n'est pas nécessairement due à une introduction.

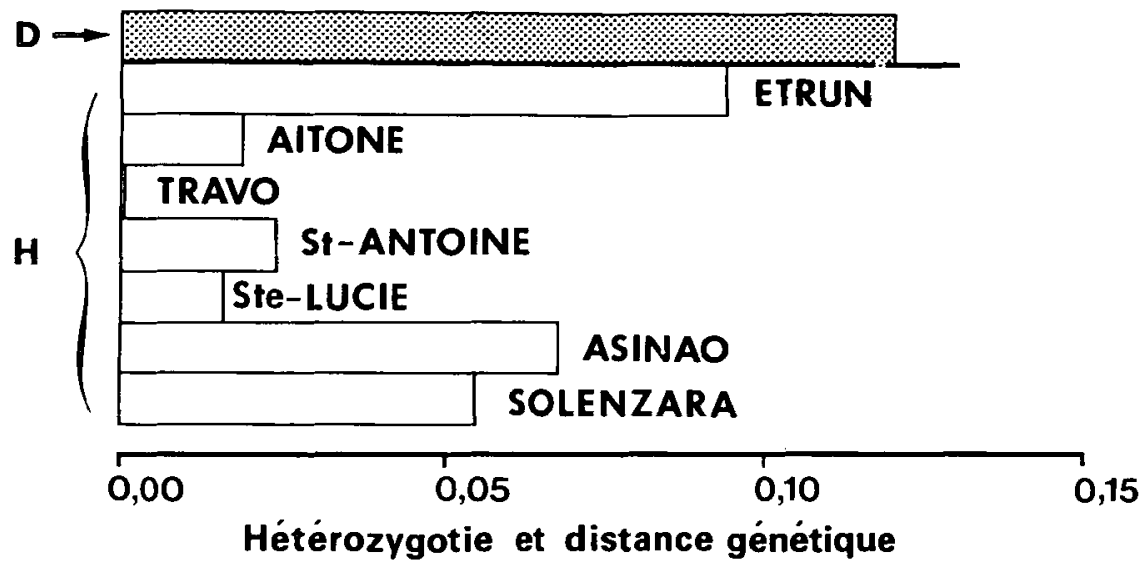

Figure 2 : Diversité génique intrapopulation, H (calculée selon NEI, 1975), dans les sept échantillons étudiés et valeur moyenne de la distance génétique standard. D (calculée selon NEI, 1975) entre échantillons domestiques et corses. La diversité génique élevée de l'échantillon d'Asinao est due à l'importante différenciation génétique entre populations corses et domestique et à la forte diversité génique (H) de cette dernière.

Figure 2 : Intrapopulation gene diversity, H (defined by NEI, 1975), of the seven samples and mean value of the standard genetic distance, $D$ (defined by NEI, 1975), between the domestic and Corsican samples. The high gene diversity in the Asinao sample is due to the large genetic differentiation between the Corsican and domesticaded populations and the high gene diversity in the latter.

Six allèles de pisciculture sont observés dans la rivière Solenzara : Aat-4 (65), Fdp-1 (100), Fum-1,2 (115+130+140), Mdh-2 (200), Pmi-2 (105) et Sdh-1 (-100). Ces six allèles représentent également des formes dérivées hautement caractéristiques des souches domestiques et de certaines populations de la zone atlantique et signalent donc l'existence d'une introduction de gènes de la souche Etrun dans le stock de la Solenzara. Dans ce cas, l'absence de Ldh-5 (100) peut être expliquée en supposant que la "contamination génétique" de la population est plus ancienne et que cet allèle a été éliminé par dérive génétique ou contre sélection.

Compte tenu de la faible taille des échantillons, les tests statistiques permettant de mettre en évidence des déséquilibres de liaison ou-des déficits d'hétérozygotes ne sont pas applicables. L'analyse des fréquences génotypiques et des associations alléliques fait cependant apparaître les faits suivants : 
- Tous les individus, à l'exception de deux, sont fixés pour un ou plusieurs allèles spécifiques des populations corses, ce sont donc des "hybrides" corses domestiques de deuxième génération ou plus. Cela signifie qu'une fraction, au moins, des descendants issus de croisement entre individus domestiques et autochtones atteignent la maturité sexuelle et produisent à leur tour des descendances viables.

- Dans l'échantilion de St-Antoine, l'un des individus est fixé pour l'allèle de pisciculture à deux locus (Agp-2 et Ldh-5) et présente un allèle de pisciculture à quatre autres locus (Cpk-1, Fum-1,2, Mdh-3 et Pmi-2). Compte tenu de la faible fréquence de ces allèles dans l'échantillon, cet événement est très improbable sous l'hypothèse de panmixie et le sujet considéré n'appartient donc pas au même pool génique que le reste de l'échantillon. Par ailleurs, il ne possède aucun allèle spécifique corse; il s'agit de toute évidence d'un poisson de repeuplement. Les fréquences géniques ont été recalculées après élimination de cet individu (tableau $n^{\circ} 1$ ). II apparaît que la fréquence de l'allèle Ldh-5 (100) est toujours inférieure à celle de Fdp-1 (100) dans les trois échantillons où ces deux locus ont pu être examinés (Solenzara, St-Antoine, Asinao), alors qu'elle lui est supérieure dans la souche de repeuplement.

- Dans l'échantillon de la Solenzara, l'allèle Mdh-2 (200), spécifique de la souche de pisciculture n'apparaît qu'à l'état homozygote, aucun individu hétérozygote porteur de cet allèle n'étant observé. La même observation peut-être faite pour Pmi-2 (105). Une telle situation est incompatible avec l'hypothèse d'une population en équilibre.

\section{DISCUSSION}

Des phénomènes d'introgression dus au repeuplement ont été clairement démontrés dans le cas de la truite arc-en-ciel et la truite à gorge coupée, Salmo clarki seleneris, (BUSACK et GALL, 1981).

A des niveaux infraspécifiques, l'existence de "contamination génétique" par repeuplement a été suggérée, plutôt que prouvée, chez la truite arc-en-ciel (ALLENFORF et al., 1980; CAMPTON et al., 1985) et la truite fario (KRIEG et GUYOMARD, 1985). Par contre, les résultats que nous présentons ici démontrent clairement l'introgression de gènes de souches de pisciculture dans le pool génique des populations naturelles corses. Cette conclusion est confirmée par les dénombrements de caeca pyloriques. Les populations corses se caractérisent par un faible nombre de caeca (SPILLMAN, 1961 ; OLIVARI et BRUN, 1984). En revanche, ce nombre est élevé chez la souche Etrun (KRIEG, 1984). Les dénombrements réalisés sur les échantillons du Rizzanèse et de la Solenzara dont nous avons analysé le polymorphisme enzymatique (OLIVARI et BRUN, résultats non publiés) font apparaître une nette relation entre la proportion de gènes domestiques introduits et le nombre moyen de coeca pyloriques (comparaison inter-échantillons). Cette "contamination" devrait également être mise en évidence par de nouveaux locus discriminants tels que Tfn fixé pour l'allèle Tfn (98) dans les populations corses et du littoral méditerranéen non soumises au repeuplement (tableau 2; GUYOMARD; résultats non publiés) et pour l'allèle Tfn (100) dans les souches de pisciculture.

Si cette contamination atteint occasionnellement des niveaux élevés, elle n'en demeure pas moins relativement faible, en moyenne, dans les rivières du Rizzanèse et de la Solenzara et la persistance des populations de truite fario dans ces deux rivières ne doit rien au repeuplement.

La mise en évidence d'une contamination génétique pose le problème du devenir des gènes introduits, toutes les évolutions étant, a priori, possibles, de l'élimination systématique, mais à des rythmes éventuellement différents, des gènes domestiques au remplacement progressif de la population naturelle d'origine. Nos résultats montrent qu'il n'y a pas d'isolement reproducteur total, en milieu naturel, entre les populations corses et la souche de repeuplement. Cependant, cela ne signifie pas que les allèles de "pisciculture" soient introduits définitivement dans ces populations naturelles. Il est possible que tout ou partie de ces gènes soient éliminés par la sélection naturelle en cas d'interruption du flux génique (arrêt des repeuplements). Ainsi, le déficit relatif de l'allèle Ldh-5 (100), qui reste à confirmer sur des échantilions plus importants, pourrait résulter d'une sélection contre cet allèle ou en faveur de Fdp-1 (100) (ou affectant des gènes qui leur sont liés). Des effets sélectifs ont été évoqués pour expliquer l'introgression différentielle de gènes nucléaires chez deux semi-espèces européennes de souris (BONHOMME, 1984). De même, le déficit d'hétérozygotes observé dans l'échantillon de la Solenzara pourrait être la conséquence de l'existence de mécanismes sélectifs internes à la population favorisant les structures homozygotes à certains locus ou fragments chromosomiques.

Compte tenu du faible nombre d'individus et d'échantillons examinés, ces hypothèses sont, pour l'instant, spéculatives et des interprétations neutralistes peuvent également être avancées pour les expliquer : Fdp-1 (100) pourrait ne pas être une forme dérivée, mais correspondre à un polymorphisme ancestral; l'excédent de Fdp- 1 (100) résulterait alors de sa présence initiale dans la population. Cette hypothèse est, cependant, peu probable car cet allèle n'apparaît dans aucun des trois échantillons peu ou pas affectés par le repeuplement (Aïtone, Travoet Ste-Lucie). II n'apparaît pas non plus dans les échantillons du littoral méditerranéen ne montrant aucune trace de repeuplement (GUYOMARD, résultats non publiés). Le déficit d'hétérozygotes de l'échantillon de la Solenzara pourrait être lié à une immigration, à partir des secteurs amont ou aval, d'individus issus de stocks 
possédant les allèles Mdh-2 (200) et Pmi-2 (105) à des fréquences élevées. Pour choisir entre les hypothèses sélectionnistes ou neutralistes proposées, une meilleure connaissance de la structure génétique des stocks, en amont et en aval des secteurs échantillonnés et sur les bassins hydrographiques avoisinants, est indispensable.

Les populations corses "contaminées" par le repeuplement et, certainement, celles du littoral méditerranéen français constituent un modèle original d'hybridation secondaire récente, offrant la possibilité d'étudier l'évolution de la structure génétique au cours des stades initiaux de mise en contact de deux formes différenciées d'une même espèce. Dans une perspective plus appliquée, elles s'avèrent, de par leur originalité génétique, extrêmement favorables à une évaluation des effets et de l'intérêt des repeuplements réalisés dans ces bassins hydrographiques.

\section{REMERCIEMENTS}

Cette étude a été réalisée dans le cadre de la convention C.S.P.-I.N.R.A. “Génétique des populations de truite commune".

Nous remercions le Parc Naturel Régional de Corse ainsi que G. BRUN et G. OLIVARI (Laboratoire d'Hydrobiologie, Université d'Aix-Marseille I) pour leur aide lors des campagnes d'échantillonnage.

\section{BIBLIOGRAPHIE}

ALLENDORF F.W., ESPELAND D.M., SCOWD.T.PHELPS S., 1980.Coexistence of native and introduced rainbow trout in Kootenai River drainage. Proceedings of the Montana Academy of Sciences, 39, 28-36.

BONHOMME F., 1984. Approche moléculaire de l'évolution d'un complexe d'espèces : variabilité génétique et polymorphisme des gènes de structure et des ADN satellites et mitochondriaux chez Mus musculus et apparentés. Thèse de doctorat d'état, Université des Sciences et Techniques du Languedoc, Montpellier.

BUSACK C. A., GALL G.A.E., 1981. Introgressive hybridization in populations of Paiute cutthroat trout (Salmo clarki seleneris). Can. J. Fish. Aquat. Sci., 94, 939-951.

CAMPTON D.E., JOHNSTON J.M., 1985. Electrophoretic evidence for a genetic admixture of the native and non-native rainbow trout in the Yakima River, Washington. Trans. Am. Fish. Soc., 114, 782-793.

CHEVASSUS B., GUYOMARD R., 1983. Recherches sur la génétique de la trujte fario et du saumon atlantique. Rapport final de la convention INRA-CSP 1980-1982, 58 pp.

GUYOMARD R., 1986. Gene segregation in gynogenetic brown trout (Salmo trütta L.) : systematically high frequencies of post reduction. Genet. Sel. Evol., (sous presse).

GUYOMARD R., KRIEG F., 1983. Electrophoretic variation in six populations of brown trout /Sa/mo trutta L.). Can. J. Genet. Cytol., 25, 403-413.

HENNIG W., 1966. Phylogenetic Systematics. Univ. Illinois Press, Urbana, 263 pp.

KRIEG F., 1984. Recherche d'une différenciation génétique entre populations de Salmo trutta. Thèse de $3^{\text {e }}$ cycle. Université de Paris-sud, Orsay.

KRIEG F., GUYOMARD R., 1983. Mise en évidence électrophorétique d'une forte différenciation génétique entre populations de truite fario de Corse. C.R. Acad. Sci., Paris, 296, 1089-1094.

KRIEG F., GUYOMARD R., 1985. Population genetics of French brown trout (Salmo trutta L.) : large geographical differentiation of wild populations and high similarity of domesticated stocks. Génét. Sél. Evol., 17 (2), 225-242.

NEI M., 1975. Molecular population genetics. North-Holland, Amsterdam and New-York, 228 pp.

OLIVARI G., BRUN G., 1984. Le nombre de caeca pyloriques dans les populations naturelles de truites en Corse. Actes du Colloque International sur les Vertébrés Terrestres et Dulçaquicoles des lles méditerranéennes (sous presse).

SPILLMAN C., 1961. Faune de France, poissons d'eau douce. Lechevallier, Paris, 291 p. 